\title{
Novel fast simulation technique for axisymmetric plasma wakefield acceleration configurations in the blowout regime
}

\author{
P. Baxevanis and G. Stupakov \\ SLAC National Accelerator Laboratory, Menlo Park, California 94025, USA
}

(Received 14 November 2017; published 24 July 2018)

\begin{abstract}
In the blowout regime of plasma wakefield acceleration (PWFA), which is the most relevant configuration for current and future applications and experiments, the plasma flow that is excited by the ultrarelativistic drive beam is highly nonlinear. Thus, fast and accurate simulation codes are indispensable tools in the study of this extremely important problem. We have developed a novel algorithm that deals with the propagation of axisymmetric bunches of otherwise arbitrary profile through a cold plasma of uniform density. In contrast to the existing PWFA simulation tools, our code PLEBS (plasmaelectron beam simulations) uses a new computational scheme which ensures that the transverse and longitudinal directions are completely decoupled - a feature which significantly enhances the speed and robustness of the new method. Our numerical results are benchmarked against the QUICKPIC code and excellent agreement is established between the two approaches. Moreover, our new technique provides a very convenient framework for studying issues such as beam loading and short-range wakefields within the plasma cavity.
\end{abstract}

DOI: 10.1103/PhysRevAccelBeams.21.071301

\section{INTRODUCTION}

The technique of plasma wakefield acceleration (PWFA), in which an intense, ultrarelativistic drive beam excites strong accelerating fields as it moves through a dense plasma column, is one of the most promising schemes for achieving the unprecedented acceleration gradients necessary for linear collider or compact freeelectron laser (FEL) applications [1,2]. Unlike the early research efforts in this area [3-5], most contemporary iterations of this concept are based on the so-called blowout regime [6,7], in which the density of the driver is comparable to (or considerably higher than) that of the plasma background. In this case, the drive beam expels plasma electrons from its path in such a way that a comoving cavity (or bubble) is created in its wake. The corresponding plasma flow is highly nonlinear, which makes the development of a rigorous, analytical theory for this regime extremely difficult. Most efforts along these lines have focused on a phenomenological treatment of the problem, using insight gained from simulations [8-10]. Recent attempts to construct an analytical description of the blowout regime from first principles have been surprisingly successful, but the resulting expressions are only applicable

Published by the American Physical Society under the terms of the Creative Commons Attribution 4.0 International license. Further distribution of this work must maintain attribution to the author(s) and the published article's title, journal citation, and DOI. in the limit of small driver charge and small driver dimensions [11]. As a result, simulation codes remain the main tool for treating the PWFA problem in its general form [12-15].

Moreover, the beam loading effect associated with the presence of a realistic witness bunch and beam instability issues related to short-range wakefields (longitudinal and transverse) induced within the plasma bubble are topics of considerable importance when assessing the feasibility of the PWFA concept [16]. In this paper, we present the outline of a novel PWFA simulation code that, apart from treating the basic problem of electron acceleration, also offers a natural and convenient framework for dealing with more advanced subjects like the ones mentioned above. Starting entirely from first principles, our semianalytical formalism is based on the assumption that we are only interested in the steady-state regime of the interaction. This quasistatic approximation implies that we neglect the longitudinal plasma nonuniformity and other fast effects associated with the injection process. Another crucial assumption is that the PWFA configuration under consideration is axially symmetric. Restricting our analysis to the case of 2D cylindrical geometry enables us to simplify our treatment while retaining most of the underlying physics. At the same time, we can readily take into account the finite size of both the driver and the witness bunches, which can have an arbitrary density profile.

Our development is organized as follows: In Sec. II, we formulate the equations for the steady-state electromagnetic field excited by a beam moving with the speed of light in a 
uniform plasma. Section III then derives the single particle equations of motion for the plasma electrons, while Sec. IV introduces some basic concepts regarding the description of the plasma flow in terms of macroparticles. The remaining details of the computational algorithm are given in Sec. V, while the results of a simple numerical study of the beam loading effect are presented in Sec. VI. As part of the latter, we also include a comparison between our code and an existing particle-in-cell (PIC) simulation tool. Our analytical derivation is completed in Sec. VII, where we show how our code can be adapted for the calculation of short-range wakefields inside the plasma cavity. Finally, Sec. VIII summarizes the main results of this paper.

\section{EQUATIONS FOR THE ELECTROMAGNETIC FIELD}

In this section, we formulate the equations that describe the plasma dynamics behind a finite-size driver moving through a cold plasma with the speed of light. To start with, we assume that the driver propagates along the $z$ axis in the positive direction. As we have already mentioned, our analysis is restricted to the steady-state case so sufficient time is assumed to have elapsed since the injection stage and the equilibrium value of the plasma electron number density $n_{0}$ is taken as uniform in space (moreover, we disregard the motion of the ions). In order to incorporate the effect of beam loading, we also assume that a finite-size, ultrarelativistic witness beam follows the driver through the plasma column at a fixed distance behind it. Both the driver and the witness bunches are assumed to be on axis, radially symmetric and nonevolving. Following the standard convention, we normalize the time $t$ to $\omega_{p}^{-1}$, length to $k_{p}^{-1}$, and velocities to the speed of light $c$. Here, $\omega_{p}=c k_{p}$ is the plasma oscillation frequency, given by $\omega_{p}=\sqrt{4 \pi n_{0} e^{2} / m}=c \sqrt{4 \pi n_{0} r_{e}}$ $\left(r_{e}=e^{2} / m c^{2}\right.$ is the classical electron radius). We also normalize momenta to $m c$, fields (electric and magnetic) to $m c \omega_{p} / e$, potentials (scalar and vector) to $m c^{2} / e$, charge densities to $n_{0} e$ and current densities to $e n_{0} c$.

Assuming that all potentials and fields depend on $z$ and $t$ solely through the combination $\xi=t-z$, which expresses the longitudinal position with respect to a fixed point of the driver, we find the following equations for the nonzero components of the (scaled) electric and magnetic fields $E_{r}$, $E_{z}$ and $B_{\theta}$ in the cylindrical coordinate system (for the current density, we use the relation $\boldsymbol{j}=-n \boldsymbol{v}$, where the negative electron charge is explicitly taken into account):

$$
\begin{aligned}
\frac{1}{r} \frac{\partial}{\partial r} r B_{\theta} & =\frac{\partial E_{z}}{\partial \xi}-n v_{z}-n_{\mathrm{ext}}, \\
\frac{\partial}{\partial \xi}\left(B_{\theta}-E_{r}\right) & =-n v_{r}, \\
\frac{\partial E_{z}}{\partial r} & =-n v_{r},
\end{aligned}
$$

where $n$ is the scaled plasma electron density, $n_{\text {ext }}=n_{d}+n_{w}$ is the total "external" density due to the driver and witness beams while $v_{r}$ and $v_{z}$ are the radial and longitudinal components of the electron collective velocity. We also need the equation for the divergence $\nabla \cdot \boldsymbol{E}$, which yields

$$
\frac{1}{r} \frac{\partial}{\partial r} r E_{r}-\frac{\partial}{\partial \xi} E_{z}=1-n-n_{\mathrm{ext}} .
$$

From Eqs. (1b) and (1c), we can introduce the pseudopotential $\psi$ such that

$$
B_{\theta}-E_{r}=\frac{\partial \psi}{\partial r}, \quad E_{z}=\frac{\partial \psi}{\partial \xi} .
$$

The function $\psi$ is the difference between the scalar potential $\phi$ and the longitudinal component of the vector potential $A_{z}$, i.e. $\psi=\phi-A_{z}$. Combining Eq. (1a) with (2), we obtain

$$
\frac{1}{r} \frac{\partial}{\partial r} r\left(B_{\theta}-E_{r}\right)=n\left(1-v_{z}\right)-1,
$$

which gives us the following equation for $\psi$ :

$$
\frac{1}{r} \frac{\partial}{\partial r} r \frac{\partial \psi}{\partial r}=n\left(1-v_{z}\right)-1 .
$$

Additionally, we have an equation for the gradient $\partial_{\xi} \psi$, namely

$$
\frac{\partial}{\partial r} \partial_{\xi} \psi=-n v_{r}
$$

We also need an equation for the azimuthal magnetic field $B_{\theta}$. To start with, we differentiate Eq. (1a) with respect to $r$ and substitute $\partial E_{z} / \partial r$ with the value extracted from Eq. (1c). This yields the relation

$$
\frac{\partial}{\partial r} \frac{1}{r} \frac{\partial}{\partial r} r B_{\theta}=-\frac{\partial}{\partial \xi} n v_{r}-\frac{\partial}{\partial r} n v_{z}-\frac{\partial n_{\mathrm{ext}}}{\partial r} .
$$

We will discuss how Eqs. (5) and (7) are solved numerically in Sec. V.

\section{EQUATIONS OF MOTION FOR THE PLASMA ELECTRONS}

In addition to the equations for the electromagnetic field derived in the previous section, we need the equations of motion for the plasma electrons. Using the relation $d \xi=$ $\left(1-v_{z}\right) d t$ for the differentials $d t$ and $d \xi$, these equations can be written as

$$
\begin{aligned}
& \frac{d p_{r}}{d t}=\left(1-v_{z}\right) \frac{d\left(\gamma v_{r}\right)}{d \xi}=-E_{r}+v_{z} B_{\theta}, \\
& \frac{d p_{z}}{d t}=\left(1-v_{z}\right) \frac{d\left(\gamma v_{z}\right)}{d \xi}=-E_{z}-v_{r} B_{\theta},
\end{aligned}
$$


where $p_{r}=\gamma v_{r}$ and $p_{z}=\gamma v_{r}$ are the radial and longitudinal components of the momentum vector (note that these are now the individual particle momenta). We also have a corresponding equation for the relativistic $\gamma$ factor:

$$
\frac{d \gamma}{d t}=\left(1-v_{z}\right) \frac{d \gamma}{d \xi}=-E_{z} v_{z}-E_{r} v_{r}
$$

There is an important integral of motion in this problem, given by $\gamma-p_{z}-\psi=$ const. This can be shown using Eqs. (8b), (9) and (3) [17]. Assuming that the plasma electrons are at rest at $\xi=\xi_{\text {init }}$, where $\xi_{\text {init }}$ is the initial (lower) $\xi$-value corresponding to the front of the driver, we obtain $\gamma-p_{z}-\psi=1$ or

$$
1-v_{z}=\frac{1}{\gamma}(1+\psi)
$$

where we have also used the fact that $\psi\left(r, \xi=\xi_{\text {init }}\right)=0$, a property which will be verified later on. From this, we can express $p_{z}$ and $\gamma$ in terms of $p_{r}$ and $\psi$ via the relations

$$
\begin{gathered}
p_{z}=\frac{1}{2(1+\psi)}\left[1+p_{r}^{2}-(1+\psi)^{2}\right], \\
\gamma=\frac{1}{2(1+\psi)}\left[1+p_{r}^{2}+(1+\psi)^{2}\right] .
\end{gathered}
$$

Next, we rewrite the equation for the radial momentum $p_{r}$ as

$$
\begin{aligned}
\frac{d p_{r}}{d \xi} & =-\frac{1}{1-v_{z}} E_{r}+\frac{v_{z}}{1-v_{z}} B_{\theta} \\
& =-\frac{1}{1-v_{z}}\left(B_{\theta}-\frac{\partial \psi}{\partial r}\right)+\frac{v_{z}}{1-v_{z}} B_{\theta} \\
& =\frac{\gamma}{1+\psi} \frac{\partial \psi}{\partial r}-B_{\theta},
\end{aligned}
$$

where we have used Eqs. (3) and (10). In addition to the above, we also require an equation for the radial orbit. Starting from $d r / d t=v_{r}$, we obtain

$$
\frac{d r}{d \xi}=\frac{v_{r}}{1-v_{z}}=\frac{p_{r}}{1+\psi} .
$$

We should point out that the usefulness of $\xi=t-z$ as an independent variable instead of the time $t$ (in the particle equations) is enhanced by the fact that the former is a monotonically increasing function of the latter (recall that $\left.d \xi / d t=1-v_{z}>0\right)$. The single-particle equations of motion should be supplemented by the continuity equation for the plasma density $n$, namely

$$
\frac{1}{r} \frac{\partial}{\partial r}\left(r n v_{r}\right)+\frac{\partial}{\partial \xi} n\left(1-v_{z}\right)=0 .
$$

\section{MACROPARTICLES}

In our computational algorithm, the plasma electrons are represented as a sum over macroparticles. Each macroparticle is characterized by the dimensionless charge $q_{i}$, the coordinate $r_{i}(\xi)$ and momenta $p_{z i}(\xi)$ and $p_{r i}(\xi)$. The coordinates and momenta satisfy the equations of motion (12) and (13), with the fields on the right-hand side of the equations taken at the positions of the particles. The plasma electron density $n$ and the corresponding currents are represented as a sum over macroparticles according to the relations

$$
\begin{aligned}
n & =\sum_{i} \frac{q_{i}}{r_{i}(\xi)\left[1-v_{z i}(\xi)\right]} \delta\left[r-r_{i}(\xi)\right], \\
n\left(1-v_{z}\right) & =\sum_{i} \frac{q_{i}}{r_{i}(\xi)} \delta\left[r-r_{i}(\xi)\right], \\
n v_{r} & =\sum_{i} \frac{q_{i} v_{r i}(\xi)}{r_{i}(\xi)\left[1-v_{z i}(\xi)\right]} \delta\left[r-r_{i}(\xi)\right],
\end{aligned}
$$

where $v_{z i}$ and $v_{r i}$ are the macroparticle velocities and the summation goes over all macroparticles in the system. The particular form of the weights in front of the delta functions in Eq. (15) is chosen in such a way that the continuity equation (14) is automatically satisfied by the expressions given above.

The weights $q_{i}$ are determined by the initial coordinates of the macroparticles, $r_{i}\left(\xi_{\text {init }}\right)$. Integrating the first equation of (15) from $r_{a}$ to $r_{b}$ (where $r_{a}$ and $r_{b}$ are arbitrary radii) with the weight $r$ and using $v_{z i}\left(\xi_{\text {init }}\right)=0$ and $n\left(r, \xi_{\text {init }}\right)=1$ (both stemming from the fact that the plasma is at rest in front of the driver) we obtain

$$
\int_{r_{a}}^{r_{b}} d r r=\frac{1}{2}\left(r_{b}^{2}-r_{a}^{2}\right)=\sum_{r_{a}<r_{i}\left(\xi_{\text {nit }}\right)<r_{b}} q_{i},
$$

where the inequality means that only those macroparticles with initial radius between $r_{a}$ and $r_{b}$ are included in the summation. To approximately satisfy this equation we choose $q_{i}=\delta r_{i} r_{i}\left(\xi_{\text {init }}\right)$ where $\delta r_{i}=r_{i+1}-r_{i}$ is the separation between the particles; then the sum on the right-hand side becomes $\sum_{i} \delta r_{i} r_{i}\left(\xi_{\text {init }}\right) \approx \frac{1}{2}\left(r_{b}^{2}-r_{a}^{2}\right)$. Of course, to minimize the error stemming from this approximation one has to use many particles with the distance between them $\delta r_{i}$ much smaller than the transverse size of the plasma flow.

\section{CALCULATION OF THE FIELDS}

In this section, we focus on the solution of the equations for the plasma electromagnetic fields (5) and (7). To start with, we decompose the magnetic field into two parts via $B_{\theta}=\bar{B}_{\theta}+B_{\theta}^{(0)}$, where $B_{\theta}^{(0)}$ is the solution of the inhomogeneous equation 


$$
\frac{\partial}{\partial r}\left[\frac{1}{r} \frac{\partial\left(r B_{\theta}^{(0)}\right)}{\partial r}\right]=-\frac{\partial n_{\mathrm{ext}}}{\partial r}
$$

This quantity, which expresses the contribution of the driver and witness beams to the total magnetic field $B_{\theta}$, is given in an analytical fashion by

$$
B_{\theta}^{(0)}(r, \xi)=-\frac{1}{r} \int_{0}^{r} d \hat{r} \hat{r} n_{\mathrm{ext}}(\hat{r}, \xi)
$$

The equation for the part of the magnetic field due to the plasma electron flow then becomes

$$
\frac{\partial}{\partial r}\left[\frac{1}{r} \frac{\partial}{\partial r} r \bar{B}_{\theta}\right]=-\frac{\partial}{\partial \xi} n v_{r}-\frac{\partial}{\partial r} n v_{z} .
$$

We need to solve this equation with the boundary conditions: $B_{\theta}=0$ at $r=0$ and $B_{\theta} \rightarrow 0$ when $r \rightarrow \infty$.

For the radial current $n v_{r}$, we use the last equation of (15), which can be written as

$$
n v_{r}=\sum_{i} \frac{q_{i} p_{r i}}{r_{i}\left(1+\psi_{i}\right)} \delta\left(r-r_{i}\right)
$$

where $\psi_{i}=\psi_{i}(\xi)=\psi\left[r_{i}(\xi), \xi\right]$ and we have also made use of Eq. (10). In order to simplify the notation, we have temporarily suppressed the $\xi$-dependence of the various macroparticle quantities. For the longitudinal plasma current $n v_{z}$, we have the relation

$$
n v_{z}=\sum_{i} \frac{q_{i} v_{z i}}{r_{i}\left(1-v_{z i}\right)} \delta\left(r-r_{i}\right)=\sum_{i} \frac{q_{i}}{r_{i}}\left(\frac{\gamma_{i}}{1+\psi_{i}}-1\right) \delta\left(r-r_{i}\right),
$$

where we have again used Eq. (10). The radial derivative $\partial\left(n v_{z}\right) / \partial r$ is easily calculated to be

$$
\frac{\partial}{\partial r} n v_{z}=\sum_{i} \frac{q_{i}}{r_{i}}\left(\frac{\gamma_{i}}{1+\psi_{i}}-1\right) \delta^{\prime}\left(r-r_{i}\right)
$$

where the prime denotes differentiation with respect to $r$. Considerably more work is required for obtaining the longitudinal derivative $\partial_{\xi}\left(n v_{r}\right)$. The end result of the corresponding calculation is

$$
\begin{aligned}
\frac{\partial}{\partial \xi} n v_{r}= & \sum_{i} \frac{q_{i}}{r_{i}}\left[\frac{\delta\left(r-r_{i}\right)}{1+\psi_{i}} \frac{d p_{r i}}{d \xi}-\frac{p_{r i} \delta^{\prime}\left(r-r_{i}\right)}{1+\psi_{i}} \frac{d r_{i}}{d \xi}-\frac{p_{r i} \delta\left(r-r_{i}\right)}{\left(1+\psi_{i}\right)^{2}}\left(\frac{d r_{i}}{d \xi}\left(\partial_{r} \psi\right)_{i}+\left(\partial_{\xi} \psi\right)_{i}\right)-\frac{p_{r i} \delta\left(r-r_{i}\right)}{r_{i}\left(1+\psi_{i}\right)} \frac{d r_{i}}{d \xi}\right] \\
= & \sum_{i} \frac{q_{i}}{r_{i}}\left[\frac{\delta\left(r-r_{i}\right)}{1+\psi_{i}}\left(\frac{\gamma_{i}}{1+\psi_{i}}\left(\partial_{r} \psi\right)_{i}-\bar{B}_{\theta}-B_{\theta}^{(0)}\left[r_{i}(\xi), \xi\right]\right)-\frac{p_{r i}^{2} \delta^{\prime}\left(r-r_{i}\right)}{\left(1+\psi_{i}\right)^{2}}\right. \\
& \left.-\frac{p_{r i} \delta\left(r-r_{i}\right)}{\left(1+\psi_{i}\right)^{2}}\left(\left(\partial_{r} \psi\right)_{i} \frac{p_{r i}}{1+\psi_{i}}+\left(\partial_{\xi} \psi\right)_{i}\right)-\frac{p_{r i}^{2} \delta\left(r-r_{i}\right)}{r_{i}\left(1+\psi_{i}\right)^{2}}\right]
\end{aligned}
$$

where use has been made of Eqs. (12) and (13). This transformation of the derivative $\partial\left(n v_{r}\right) / \partial \xi$ can also be found in Ref. [18].

Substituting Eqs. (22) and (23) into Eq. (19) for $\bar{B}_{\theta}$, we observe that on the right-hand side there is a sum of terms each of which is either proportional to $\delta\left(r-r_{i}\right)$ or $\delta^{\prime}\left(r-r_{i}\right)$. For $r_{i}<r<r_{i+1}$ (where we have tacitly assumed that the macroparticles have been labeled according to increasing radius), the plasma currents are zero and $\bar{B}_{\theta}$ satisfies the relation $\partial_{r}\left[(1 / r) \partial_{r}\left(r \bar{B}_{\theta}\right)\right]=0$. Thus, the magnetic field between the delta functions with labels $i$ and $i+1$ can be represented as

$$
\bar{B}_{\theta}=a_{i} r+\frac{b_{i}}{r}
$$

where $a_{i}$ and $b_{i}$ are functions of $\xi$. Our objective now becomes to find the corresponding matching conditions at the locations of the delta functions, $r=r_{i}(\xi)$. These conditions are derived in the Appendix and are formulated as a system of linear equations for $a_{i}, b_{i}$ :

$$
\begin{aligned}
\left(a_{i}-a_{i-1}\right) r_{i}+\frac{b_{i}-b_{i-1}}{r_{i}}= & C_{i}, \\
a_{i}-a_{i-1}-\frac{b_{i}-b_{i-1}}{r_{i}^{2}}= & A_{i}\left(a_{i-1} r_{i}+\frac{b_{i-1}}{r_{i}}\right) \\
& +B_{i}-\frac{C_{i}}{r_{i}}+\frac{1}{2} A_{i} C_{i},
\end{aligned}
$$

where the coefficients $A_{i}, B_{i}$ and $C_{i}$ are

$$
\begin{aligned}
A_{i}= & \frac{q_{i}}{r_{i}} \frac{1}{1+\psi_{i}}, \\
B_{i}= & -\frac{q_{i}}{r_{i}} \frac{\gamma_{i}}{\left(1+\psi_{i}\right)^{2}}\left(\partial_{r} \psi\right)_{i}+\frac{q_{i}}{r_{i}} \frac{p_{r i}^{2}}{\left(1+\psi_{i}\right)^{3}}\left(\partial_{r} \psi\right)_{i} \\
& +\frac{q_{i}}{r_{i}} \frac{p_{r i}}{\left(1+\psi_{i}\right)^{2}}\left(\partial_{\xi} \psi\right)_{i}+\frac{q_{i} p_{r i}^{2}}{r_{i}^{2}\left(1+\psi_{i}\right)^{2}}+\frac{q_{i}}{r_{i}} \frac{B_{\theta}^{(0)}\left(r_{i}(\xi), \xi\right)}{1+\psi_{i}}, \\
C_{i}= & \frac{q_{i}}{r_{i}} \frac{p_{r i}^{2}}{\left(1+\psi_{i}\right)^{2}}-\frac{q_{i}}{r_{i}}\left(\frac{\gamma_{i}}{1+\psi_{i}}-1\right) .
\end{aligned}
$$


Equation (25) can also be written in matrix form:

$$
\begin{aligned}
\left(\begin{array}{c}
a_{i} \\
b_{i}
\end{array}\right)= & \left(\begin{array}{cc}
1+\frac{1}{2} A_{i} r_{i} & \frac{1}{2 r_{i}} A_{i} \\
-\frac{1}{2} A_{i} r_{i}^{3} & 1-\frac{1}{2} A_{i} r_{i}
\end{array}\right)\left(\begin{array}{c}
a_{i-1} \\
b_{i-1}
\end{array}\right) \\
& +\left(\begin{array}{c}
\frac{1}{4}\left(2 B_{i}+A_{i} C_{i}\right) \\
\frac{1}{4} r_{i}\left(4 C_{i}-2 B_{i} r_{i}-A_{i} C_{i} r_{i}\right)
\end{array}\right) .
\end{aligned}
$$

Applying the recursion rule of Eq. (27) for $1 \leq i \leq N$ (where $N$ is the number of simulation particles), we can obtain the magnetic field pattern at a given $\xi$ given the pseudopotential $\psi$ and the macroparticle positions and momenta. Apart from the condition $b_{0}=0$ (which avoids a singularity at $r=0$ and guarantees that $B_{\theta}=0$ at $r=0$ ), we typically assume that $a_{N}=0$, which ensures a $1 / r$ field decay after the last particle, and hence $B_{\theta} \rightarrow 0$ when $r \rightarrow \infty$.

To complete the electrodynamics-related part of our derivation, we now consider Eq. (5). The boundary conditions for this equation are: $\psi$ is finite at $r=0$ and $\psi \rightarrow 0$ when $r \rightarrow \infty$. Substituting the second equation of (15) to the right-hand side of Eq. (5), we see that it contains terms that are proportional to $\delta\left(r-r_{i}\right)$. For the region between two neighboring macroparticles $\left(r_{i}<r<r_{i+1}\right)$, we have $(1 / r) \partial_{r}\left(r \partial_{r} \psi\right)=-1$. This relation yields a vacuum solution of the form $\psi=\bar{a}_{i}+\bar{b}_{i} \ln r-r^{2} / 4$, where $\bar{a}_{i}, \bar{b}_{i}$ are functions of $\xi$. To find relations between the adjacent coefficients $\bar{a}_{i}, \bar{b}_{i}$, we integrate Eq. (5) over the radius $r$ from $r_{i}^{-}=r_{i}-0$ to $r_{i}^{+}=r_{i}+0$ through the delta function $\delta\left(r-r_{i}\right)$. We obtain the following matching conditions for the pseudopotential $\psi$,

$$
\begin{aligned}
\partial_{r} \psi\left(r_{i}^{+}, \xi\right)-\partial_{r} \psi\left(r_{i}^{-}, \xi\right) & =\frac{q_{i}}{r_{i}(\xi)}, \\
\psi\left(r_{i}^{+}, \xi\right)-\psi\left(r_{i}^{-}, \xi\right) & =0 .
\end{aligned}
$$

With these matching conditions, we find that $\bar{a}_{i}+\bar{b}_{i} \ln r_{i}=$ $\bar{a}_{i-1}+\bar{b}_{i-1} \ln r_{i}$ and $\bar{b}_{i}-\bar{b}_{i-1}=q_{i}$. For $0<r<r_{1}$ (i.e. for radii smaller than that of the first macroparticle), we have $\psi=\bar{a}_{0}-r^{2} / 4$ since $\bar{b}_{0}$ must be equal to zero in order to avoid a singularity at $r=0$. Using the above, we can add potential terms over successive delta functions in order to construct the solution for $\psi$. The end result is $\psi(r, \xi)=\psi(0, \xi)+\Delta \psi(r, \xi)$, where

$$
\Delta \psi(r, \xi)=\sum_{r_{i}(\xi)<r} q_{i} \ln \left[\frac{r}{r_{i}(\xi)}\right]-\frac{r^{2}}{4}
$$

and

$\psi(0, \xi)=\bar{a}_{0}=-\Delta \psi\left[r_{N}(\xi), \xi\right]=-\sum_{i} q_{i} \ln \left[\frac{r_{N}(\xi)}{r_{i}(\xi)}\right]+\frac{r_{N}^{2}(\xi)}{4}$.
Here, we have adopted the condition that the pseudopotential $\psi$ is always zero at the location of the last macroparticle, i.e. $\psi\left[r_{N}(\xi), \xi\right]=0$. For a large enough radial coordinate of the last electron in the system, $r_{N}(\xi)$, this condition approximates the boundary condition $\psi \rightarrow 0$ when $r \rightarrow \infty$. From Eqs. (29) and (30), one can easily derive the partial derivatives of $\psi$ that are needed for evaluating the $A_{i}, B_{i}$, $C_{i}$ coefficients discussed earlier. In particular, we have

$$
\partial_{r} \psi(r, \xi)=\partial_{r} \Delta \psi(r, \xi)=\frac{1}{r} \sum_{r_{i}(\xi)<r} q_{i}-\frac{r}{2}
$$

for the radial derivative and $\partial_{\xi} \psi(r, \xi)=\partial_{\xi} \Delta \psi(r, \xi)-$ $\partial_{\xi} \Delta \psi\left[r_{N}(\xi), \xi\right]$ for its longitudinal counterpart, where

$$
\partial_{\xi} \Delta \psi(r, \xi)=-\sum_{r_{i}(\xi)<r} \frac{q_{i}}{r_{i}} \frac{p_{r i}}{1+\psi_{i}},
$$

and we have assumed that $d r_{N}(\xi) / d \xi=0$. Incidentally, it is worth mentioning that one of the $\xi$ derivatives mentioned above is directly related to the calculation of the on-axis longitudinal electric field, one of the main figures of merit for the PWFA. Specifically, the force per unit charge for an on-axis electron is given by $F_{z} / e=-E_{z}(0, \xi)=-\partial_{\xi} \psi(0, \xi)=\partial_{\xi} \Delta \psi\left(r_{N}, \xi\right)$, a result which is particularly useful for numerical calculations. Lastly, we point out that combining Eqs. (16) and (31) yields $\partial_{r} \psi\left(r, \xi_{\text {init }}\right)=0$, which means that $\psi\left(r, \xi_{\text {init }}\right)=$ $\psi\left(r_{N}, \xi_{\text {init }}\right)=0$. This verifies our earlier claim that the pseudopotential is zero for all $r$ at the front of the driver.

Equations (18), (24), (27) and (26), along with Eqs. (29)-(32), form the basic results of our field analysis. Their main feature is that a knowledge of the macroparticle positions and momenta at a given $\xi$ suffices for a complete description of the electromagnetic fields at that particular (relative) longitudinal position. This is also reflected in the absence of any derivatives with respect to $\xi$ on the lhs of Eqs. (19) and (5), a property which prevents the coupling of different $\xi$-values. As a result, one can readily make use of the single-particle equations of motion [i.e. Eqs. (11)-(13)] in order to propagate the particle positions and momenta to $\xi+\delta \xi$. To avoid any confusion, we rewrite the latter in our updated (macroparticle) notation as

$$
\frac{d p_{r i}}{d \xi}=\frac{\gamma_{i}}{1+\psi_{i}}\left(\partial_{r} \psi\right)_{i}-\bar{B}_{\theta}\left(r_{i}, \xi\right)-B_{\theta}^{(0)}\left(r_{i}, \xi\right), \quad \frac{d r_{i}}{d \xi}=\frac{p_{r i}}{1+\psi_{i}}
$$

and

$$
\begin{aligned}
p_{z i} & =\frac{1}{2\left(1+\psi_{i}\right)}\left[1+p_{r i}^{2}-\left(1+\psi_{i}\right)^{2}\right], \\
\gamma_{i} & =\frac{1}{2\left(1+\psi_{i}\right)}\left[1+p_{r i}^{2}+\left(1+\psi_{i}\right)^{2}\right] .
\end{aligned}
$$


In our algorithm, we use a fourth-order Runge-Kutta technique to numerically integrate the equations of motion for the plasma electrons. Another important point is that, when calculating discontinuous quantities like $\bar{B}_{\theta}, \partial_{r} \psi$ or $\partial_{\xi} \psi$ at the location of a macroparticle (i.e. at the actual location of the discontinuity), we use the arithmetic mean of the two limiting values. This is simply due to the fact that the simulation macroparticles really represent charged sheets formed by the nonlinear plasma flow. In any event, knowing the new positions and momenta enables us to calculate the fields at the updated location. Thus, a full solution of the steady-state PWFA problem (including details such as the shape of the cavity) can be constructed through this systematic, step-by-step process.

We have implemented our algorithm in the computer code PLEBS (plasma-electron beam simulations) using the MATLAB programming environment. In the next section, we will compare the results obtained with this code against QUICKPIC simulations.

\section{BEAM LOADING STUDY}

So far, we have kept the density profiles of the driver and witness beams entirely general. Here, we choose to specialize to the case where both beams have a Gaussian profile in the transverse and longitudinal directions. In doing so, we find it most convenient to start from the original quantities and then switch to their dimensionless counterparts. To begin with, we assume that the density of the drive beam is given by

$$
n_{d}(r, \xi)=n_{d 0} \exp \left(-\frac{r^{2}}{2 \sigma_{r d}^{2}}\right) \exp \left(-\frac{\left(\xi-\xi_{d}\right)^{2}}{2 \sigma_{\xi d}^{2}}\right)
$$

where $n_{d 0}$ is the peak volume (or number) density, $\sigma_{r d}$ and $\sigma_{\xi d}$ are (respectively) the transverse and longitudinal rms beam sizes and $\xi_{d}$ is the location of the drive beam centroid. Regarding the origin of $\xi$, we select $\xi_{\text {init }}=0$ and require this initial value to correspond to the front (or head) of the drive beam. This, in turn, is virtually guaranteed if we choose $\xi_{d}=N_{0} \sigma_{\xi d}$, where $N_{0} \geq 3$. The current profile of the drive beam is

$$
\begin{aligned}
I_{d}(\xi) & =c e \int n_{d}(r, \xi) 2 \pi r d r \\
& =I_{d 0} \exp \left(-\frac{\left(\xi-\xi_{d}\right)^{2}}{2 \sigma_{\xi d}^{2}}\right),
\end{aligned}
$$

where $I_{d 0}=2 \pi c e n_{d 0} \sigma_{r d}^{2}$ is the peak current. The total charge contained in the drive beam is $Q_{d}=e N_{d}=\sqrt{2 \pi} I_{d 0}\left(\sigma_{\xi d} / c\right)$, where $N_{d}=n_{d 0}(2 \pi)^{3 / 2} \sigma_{r d}^{2} \sigma_{\xi d}$ is the total number of electrons. As far as the witness beam is concerned, we have an analogous Gaussian expression, namely

$$
n_{w}(r, \xi)=n_{w 0} \exp \left(-\frac{r^{2}}{2 \sigma_{r w}^{2}}\right) \exp \left(-\frac{\left(\xi-\xi_{w}\right)^{2}}{2 \sigma_{\xi w}^{2}}\right) .
$$

The corresponding current profile is given by

$$
I_{w}(\xi)=I_{w 0} \exp \left(-\frac{\left(\xi-\xi_{w}\right)^{2}}{2 \sigma_{\xi w}^{2}}\right)
$$

with $I_{w 0}=2 \pi c e n_{w 0} \sigma_{r w}^{2}, Q_{w}=e N_{w}=\sqrt{2 \pi} I_{w 0}\left(\sigma_{\xi w} / c\right)$ etc. At this point, we switch back to the dimensionless variables employed in most of this text (we recall that volume densities are scaled with respect to the equilibrium plasma density $n_{0}$, while lengths are normalized with respect to the plasma skin depth $k_{p}^{-1}$ ). The scaled drive beam density is

$$
\tilde{n}_{d}=\tilde{n}_{d 0} \exp \left(-\frac{\tilde{r}^{2}}{2 \tilde{\sigma}_{r d}^{2}}\right) \exp \left(-\frac{\left(\tilde{\xi}-\tilde{\xi}_{d}\right)^{2}}{2 \tilde{\sigma}_{\xi d}^{2}}\right),
$$

where the tildes (temporarily) denote the scaled notation and $\tilde{n}_{d 0}$ is given by $\tilde{n}_{d 0}=\sqrt{2 / \pi} \nu_{d} /\left(\tilde{\sigma}_{r d}^{2} \tilde{\sigma}_{\xi d}\right)$. Here, $\nu_{d} \equiv$ $N_{d} r_{e} k_{p}$ is the dimensionless charge of the driver. This important quantity can be shown to be proportional to the ratio of the number of particles in the beam to the number of plasma particles within a sphere of radius $k_{p}^{-1}$ [11]. Needless to say, an entirely analogous expression can be obtained for the witness beam.

Dropping the tildes from now on, we use the above results and Eq. (18) in order to determine the external field $B_{\theta}^{(0)}$ for our case. The resulting analytical expression is

$$
\begin{aligned}
B_{\theta}^{(0)}(r, \xi)= & -n_{d 0} \exp \left(-\frac{\left(\xi-\xi_{d}\right)^{2}}{2 \sigma_{\xi d}^{2}}\right) \frac{\sigma_{r d}^{2}}{r}\left[1-\exp \left(-\frac{r^{2}}{2 \sigma_{r d}^{2}}\right)\right] \\
& -n_{w 0} \exp \left(-\frac{\left(\xi-\xi_{w}\right)^{2}}{2 \sigma_{\xi w}^{2}}\right) \frac{\sigma_{r w}^{2}}{r}\left[1-\exp \left(-\frac{r^{2}}{2 \sigma_{r w}^{2}}\right)\right] .
\end{aligned}
$$

With the above relation, we have everything that we need in order to study the beam loading effect for a Gaussian drive/witness beam configuration. For the drive beam, the parameters we have used are $\sigma_{r d}=2 \mu \mathrm{m}, \sigma_{\xi d}=20 \mu \mathrm{m}$, $I_{d 0}=2 \mathrm{kA}$ (so that $Q_{d}=334 \mathrm{pC}$ ) and $\xi_{d}=3 \sigma_{\xi d}$. As far as the witness beam is concerned, we assume that $\sigma_{r w}=2 \mu \mathrm{m}, \quad \sigma_{\xi w}=6 \mu \mathrm{m}, \quad \xi_{w}=130 \mu \mathrm{m} \quad$ and $\quad I_{w 0}=$ $2.125 / 2.55 \mathrm{kA}$ (we have examined two different cases, corresponding to a charge of $106 / 127 \mathrm{pC}$ ). The plasma density $n_{0}$ is taken to be $7 \times 10^{16} \mathrm{~cm}^{-3}$, which leads to a skin depth of $k_{p}^{-1}=20 \mu \mathrm{m}$. For these parameters, the dimensionless charge of the drive beam is $\nu_{d}=0.293$, while that of the witness bunch is $\nu_{w}=0.09 / 0.11$. Moreover, we have $n_{d 0} \approx 23.7$ and $n_{w 0} \approx 25.2 / 30.2$. The fact that these scaled density values are much larger than 

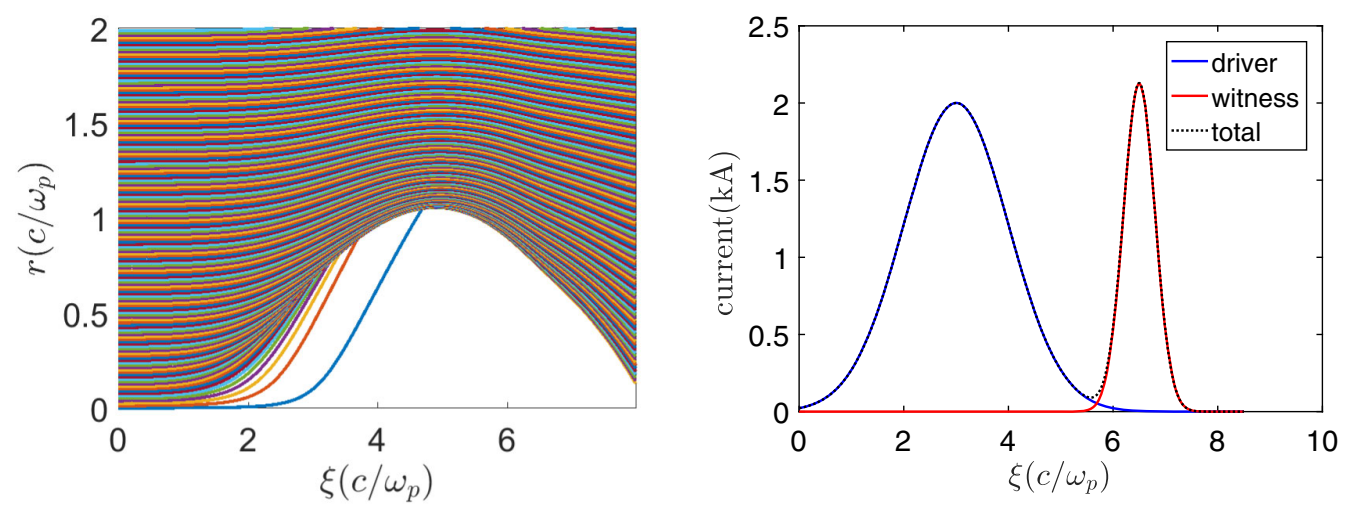

FIG. 1. Right figure: Current profiles of the Gaussian drive and witness beams. Left figure: Electron trajectories in the $r-\xi$ plane. The formation of a plasma cavity (or bubble) is evident.

unity is a typical characteristic of the blowout regime of the PWFA.

The current profiles for the drive and witness bunches are illustrated in the right graph of Fig. 1 (for a witness current of $2.125 \mathrm{kA}$ ). In the left graph of the same figure, we plot a large number of electron trajectories whose initial radii $r_{i}(\xi=0)$ are uniformly spaced on the $r$-axis. More specifically, our simulation run makes use of 2000 macroparticles $\left(N=2 \times 10^{3}\right)$ with $0<r_{i}(\xi=0)<5$, though we only plot 400 representative trajectories. The formation of a bubble is evident, with a maximum radius $r_{\text {cav }} \approx 1$ (about $20 \mu \mathrm{m}$ ) and a length $\xi_{\text {cav }} \approx 8$ (or $160 \mu \mathrm{m}$ ). In Fig. 2, we plot the on-axis, longitudinal electric field $E_{z}(0, \xi)$ as a function of $\xi$ for the two cases of witness beam current. The solid lines correspond to results from PLEBS and the dotted lines represent data from QUiCKPIC [19], a popular 3D particle-in-cell PWFA simulation tool. The simulation parameters for QUICKPIC are as follows: the grid numbers in the $x, y$ and $\xi$ directions are all equal to $2^{10}=1024$ (spanning a cubic box with size $\Delta x=\Delta y=\Delta \xi=8.35$ ) and four particles per cell are used to simulate the plasma electrons. Thus we have 1024 subdivisions in terms of $\xi$ (similar to the 750-800 integration steps we typically use in our Runge-Kutta scheme) and $2^{22} \approx 4 \times 10^{6}$ plasma particles per $\xi$-slice. The latter is much larger than the number of radial macroparticles in our code (2000) since QUICKPIC is an inherently three-dimensional algorithm that can simulate transversely asymmetrical configurations. The left graph of Fig. 2 shows the comparison for the full range of $\xi$ (up to the bubble collapse, which occurs slightly after $\xi=8$ ), while the right graph zooms in on the location of the witness bunch (witness centroid at $\xi=6.5$ ). According to a well-known pattern, the electric field is initially decelerating (for electrons) but changes sign as one moves further away from the driver. Moreover, the beam loading effect only influences the field in the neighborhood of the witness beam so that the blue and red curves, corresponding to different witness currents, are appreciably different only when $\xi>6$ ). Overall, very good agreement is observed between the two approaches, while our technique compares favorably to QUICKPIC even in terms of computation speed. In particular, our MATLAB-based code only takes a few minutes (about 3-4) to run on a simple desktop environment using a machine with 4-6 cores, whereas
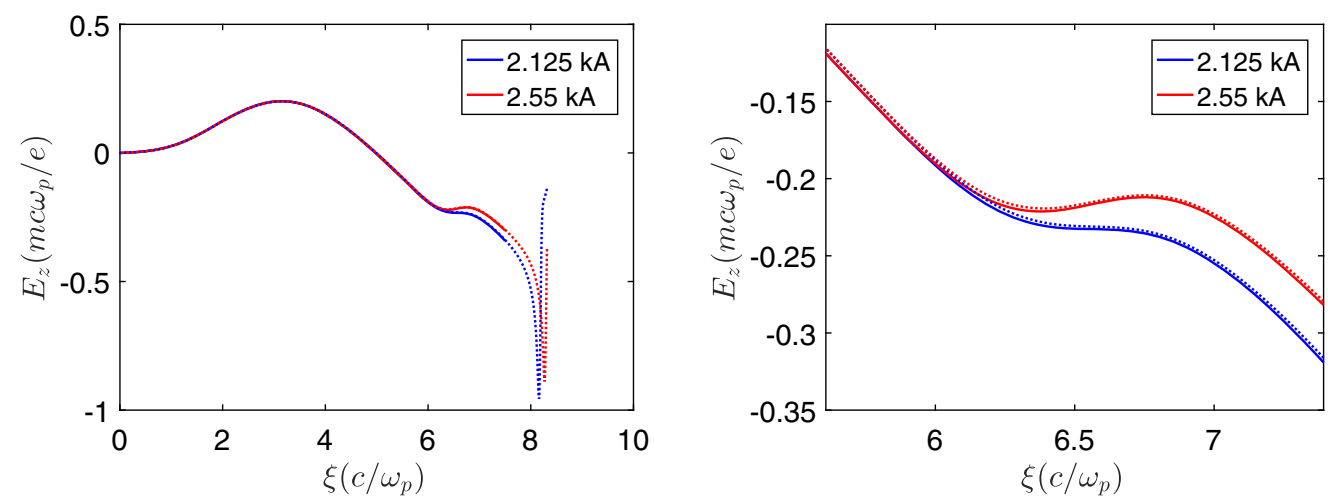

FIG. 2. On-axis longitudinal electric field $E_{z}(0, \xi)$ as a function of $\xi$ (scaled units). We show the results from our code (solid lines) versus QUickPIC data (dotted lines) for two different values of the witness beam current. The right figure focuses on the immediate neighborhood of the witness beam, while the left figure shows the full range of $\xi$. For the left figure, we intentionally use a shorter $\xi$-range for our results in order to visualize the QUICKPIC data more clearly. 


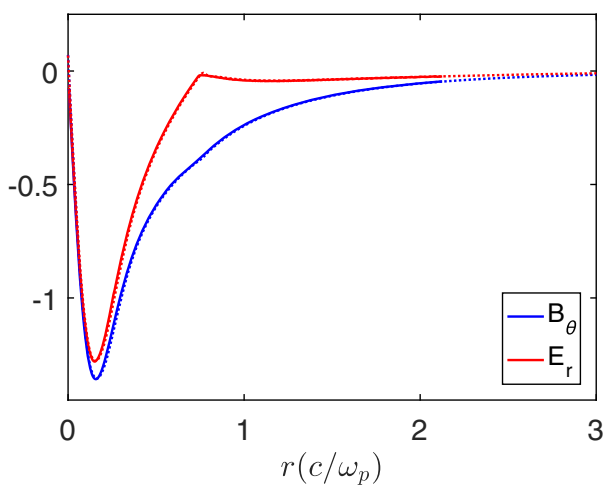

FIG. 3. Transverse field components $B_{\theta}$ and $E_{r}$ (in units of $\left.m c \omega_{p} / e\right)$ at $\xi=\xi_{w}=6.5$ as functions of $r$. The solid lines show results from our code (PLEBS) while the dotted lines show QUICKPIC data for a witness current of $2.55 \mathrm{kA}$. Since QUICKPIC is a $3 \mathrm{D}$ code utilizing a Cartesian transverse grid, the values for $B_{\theta}$ and $E_{r}$ have been obtained using the components $B_{y}$ and $E_{x}$ (respectively) at the $y=0$ plane. A slope discontinuity at $r \approx$ 0.75 corresponds to the bubble boundary (see Fig. 1, left plot). PLEBS data for $r>2.15$ are omitted in order to highlight the QUICKPIC results.

QUICKPIC requires a parallel setup with 128 cores in order to achieve a similar run time (about 6 minutes). This relatively competitive performance is largely due to the cylindrical symmetry of the case under study, which enables us to use far fewer plasma macroparticles than QUickPIC. The latter code also relies on macroparticles in order to simulate the driver and witness bunches, even when their evolution due to the interaction with the plasma is disregarded (as is the case with the results shown in this paper). Finally, Fig. 3 plots the transverse field components $B_{\theta}$ and $E_{r}$ at $\xi=\xi_{w}=6.5$ (i.e. at the location of the witness beam centroid) as functions of the radius $r$. Such graphs help one to obtain a more complete picture of the spatial distribution of the fields while still allowing for a detailed comparison with QUICKPIC. Once again, such a comparison confirms the validity of our results.

\section{WAKEFIELD CALCULATION}

In this section, our aim is to demonstrate that the algorithm which was described in the previous parts of this paper can also be used to calculate the short-range wakefields induced within the plasma bubble. As has been mentioned in the Introduction, this topic can be crucial when one seeks to evaluate a PWFA-based scheme from a stability point of view [16]. For the basic definitions, notation and analytical results regarding the topic of wakefields in a PWFA context, we rely on work presented elsewhere [20], endeavoring - at the same time-to provide a rigorous and detailed framework for the numerical calculations shown therein. To start with, we turn to the problem of the wakefields excited by an on-axis point charge $q$ located inside an axisymmetric plasma cavity at the longitudinal position $\xi=\xi_{0}$. Our goal is to calculate the longitudinal wakefield immediately behind the charge, at $\xi=\xi_{0}^{+}$. We denote by $\tilde{E}_{r}(r, \xi), \tilde{E}_{z}(r, \xi)$ and $\tilde{B}_{\theta}(r, \xi)$ the components of the modified field in the presence of the point charge $q$. As is shown in [20], the transverse field components can be expressed as

$\tilde{E}_{r}=E_{r}(r, \xi)+\Delta E_{r}(r, \xi) h\left(\xi-\xi_{0}\right)-D\left(r, \xi_{0}\right) \delta\left(\xi-\xi_{0}\right)$,

$\tilde{B}_{\theta}=B_{\theta}(r, \xi)+\Delta B_{\theta}(r, \xi) h\left(\xi-\xi_{0}\right)-D\left(r, \xi_{0}\right) \delta\left(\xi-\xi_{0}\right)$,

where $E_{r}, E_{z}$ and $B_{\theta}$ are the original components, $h(\xi)$ is the step function equal to one for positive arguments and zero otherwise, $\Delta E_{r}$ and $\Delta B_{\theta}$ denote the change of the field due to the charge $q$, and the terms with the delta function represent a shock wave characterized by the radial profile $D\left(r, \xi_{0}\right)$. The latter quantity satisfies the relation

$$
\frac{\partial}{\partial r}\left[\frac{1}{r} \frac{\partial}{\partial r} r D\left(r, \xi_{0}\right)\right]=\frac{n\left(r, \xi_{0}\right)}{\gamma\left(r, \xi_{0}\right)} D\left(r, \xi_{0}\right) .
$$

The longitudinal electric field $E_{z}$ also exhibits a discontinuity, which is expressed by

$$
\tilde{E}_{z}(r, \xi)=E_{z}(r, \xi)+\Delta E_{z}(r, \xi) h\left(\xi-\xi_{0}\right) .
$$

The jump $\Delta E_{z}(r, \xi)$ represents the longitudinal wake generated by the charge $q$ immediately behind it. It can be related to the $D$-function by means of the relation

$$
\Delta E_{z}\left(r, \xi_{0}\right)=-\frac{1}{r} \frac{\partial}{\partial r} r D\left(r, \xi_{0}\right) .
$$

Thus, calculating the longitudinal wakefield involves solving Eq. (42) for a given (that is, known from a previous calculation) plasma electron density $n(r, \xi)$. Using the first equation of (15), we can rewrite (42) as

$$
\begin{aligned}
\frac{\partial}{\partial r} \frac{1}{r} \frac{\partial}{\partial r} r D & =D \sum_{i} \frac{q_{i}}{r_{i} \gamma_{i}\left(1-v_{z i}\right)} \delta\left(r-r_{i}\right) \\
& =D \sum_{i} A_{i} \delta\left(r-r_{i}\right),
\end{aligned}
$$

where $A_{i}$ has been defined in Eq. (26) and we have used Eq. (10). The above equation can be solved using an iterative technique analogous to the one we employed in solving Eq. (19) (we note the similarity between these two equations, which have essentially identical left-hand sides). Specifically, since the rhs of (45) is zero in the intervals $r_{i}<r<r_{i+1}$ (for $i=1, \ldots, N-1$ ), we seek solutions of the form $D=\hat{a}_{i} r+\hat{b}_{i} / r$, where $\hat{a}_{i}, \hat{b}_{i}$ only depend on $\xi$. Furthermore, we have $D=\hat{a}_{0} r+2 \nu_{q} / r$ for $r<r_{1}$ and 
$D=\hat{b}_{N} / r$ for $r>r_{N}$. Here, $\nu_{q}=-q r_{e} k_{p} / e$ is a scaled charge parameter and we have also taken into account the asymptotic behavior of the shocklike field induced by a point charge in a uniform plasma $\left(E_{r} \propto \nu_{q} / r\right.$ for $r \rightarrow 0$, according to [21]). Lastly, our choice of $\hat{a}_{N}=0$ ensures that $D \rightarrow 0$ at $r \rightarrow \infty$. At the location of each delta function, we have the matching conditions of continuity and derivative jump for $D$, given by

$$
\begin{aligned}
D\left(r_{i}^{+}, \xi\right) & =D\left(r_{i}^{-}, \xi\right), \\
\partial_{r} D\left(r_{i}^{+}, \xi\right)-\partial_{r} D\left(r_{i}^{-}, \xi\right) & =A_{i} D\left(r_{i}^{-}, \xi\right) .
\end{aligned}
$$

These conditions yield the relations

$$
\begin{aligned}
\hat{a}_{i} r_{i}+\frac{\hat{b}_{i}}{r_{i}} & =\hat{a}_{i-1} r_{i}+\frac{\hat{b}_{i-1}}{r_{i}}, \\
\hat{a}_{i}-\frac{\hat{b}_{i}}{r_{i}^{2}} & =\hat{a}_{i-1}-\frac{\hat{b}_{i-1}}{r_{i}^{2}}+A_{i}\left(\hat{a}_{i-1} r_{i}+\frac{\hat{b}_{i-1}}{r_{i}}\right), \quad i=1, \ldots, N
\end{aligned}
$$

with $\hat{b}_{0}=2 \nu_{q}$ and $\hat{a}_{N}=0$. In matrix notation, the solution of this system is

$$
\left(\begin{array}{c}
\hat{a}_{i} \\
\hat{b}_{i}
\end{array}\right)=\hat{M}_{i}\left(\begin{array}{c}
\hat{a}_{i-1} \\
\hat{b}_{i-1}
\end{array}\right)
$$

where the transfer matrix $\hat{M}_{i}$ is given by

$$
\hat{M}_{i}=\left(\begin{array}{cc}
1+\frac{1}{2} A_{i} r_{i} & \frac{1}{2 r_{i}} A_{i} \\
-\frac{1}{2} A_{i} r_{i}^{3} & 1-\frac{1}{2} A_{i} r_{i}
\end{array}\right) .
$$

We note that this particular matrix is also present in Eq. (27). Combining these manipulations, we obtain

$$
\left(\begin{array}{c}
\hat{a}_{N} \\
\hat{b}_{N}
\end{array}\right)=\hat{M}\left(\begin{array}{l}
\hat{a}_{0} \\
\hat{b}_{0}
\end{array}\right)=\left(\begin{array}{ll}
\hat{M}_{11} & \hat{M}_{12} \\
\hat{M}_{21} & \hat{M}_{22}
\end{array}\right)\left(\begin{array}{c}
\hat{a}_{0} \\
\hat{b}_{0}
\end{array}\right),
$$

where $\hat{M}=\hat{M}_{N} \hat{M}_{N-1} \cdots \hat{M}_{1}$ is a cumulative matrix. Recalling that $\hat{b}_{0}=2 \nu_{q}$ and $\hat{a}_{N}=0$, we obtain $\hat{a}_{0}=$ $-2 \nu_{q} \hat{M}_{12} / \hat{M}_{11}$. Combining Eq. (44) with the small-radius expression for $D\left(D=\hat{a}_{0} r+2 \nu_{q} / r\right)$, we find that the onaxis value of the longitudinal wakefield is given by

$$
\Delta E_{z}(0, \xi)=-2 \hat{a}_{0}=\frac{4 \nu_{q} \hat{M}_{12}}{\hat{M}_{11}} .
$$

For the transverse wakefield calculation, the configuration of the leading point charge is somewhat different. In particular, the point charge $q$ is now of axis, moving with a transverse offset $\boldsymbol{a}$ inside the plasma cavity (we assume $\left.|\boldsymbol{a}| \ll k_{p}^{-1}\right)$. This time, the perturbed electromagnetic field, which now lacks axial symmetry, is given by

$$
\begin{aligned}
\tilde{\boldsymbol{E}}_{\perp} & =\boldsymbol{E}_{\perp}+\Delta \boldsymbol{E}_{\perp}(x, y, \xi) h\left(\xi-\xi_{0}\right)-\boldsymbol{D}(x, y, \xi) \delta\left(\xi-\xi_{0}\right) \\
\tilde{\boldsymbol{B}}_{\perp} & =\boldsymbol{B}_{\perp}+\Delta \boldsymbol{B}_{\perp}(x, y, \xi) h\left(\xi-\xi_{0}\right)-\hat{z} \times \boldsymbol{D}(x, y, \xi) \delta\left(\xi-\xi_{0}\right) \\
\hat{E}_{z} & =E_{z}+\Delta E_{z} h\left(\xi-\xi_{0}\right) .
\end{aligned}
$$

Assuming that $\boldsymbol{a}=a \hat{x}$, the components of the shock profile $\boldsymbol{D}$ (in cylindrical coordinates) are

$$
\left(\begin{array}{c}
D_{r} \\
D_{\theta}
\end{array}\right)=u(r, \xi)\left(\begin{array}{c}
\cos \theta \\
\sin \theta
\end{array}\right)
$$

where the radial profile $u(r, \xi)$ satisfies the relation

$$
\frac{\partial^{2} u}{\partial r^{2}}+\frac{1}{r} \frac{\partial u}{\partial r}-\frac{4 u}{r^{2}}=u \frac{n(r, \xi)}{\gamma(r, \xi)} .
$$

Our objective is to solve this equation using our matrix technique. Substituting the expression for $n$ from Eq. (15), we can rewrite (54) as

$$
\frac{\partial^{2} u}{\partial r^{2}}+\frac{1}{r} \frac{\partial u}{\partial r}-\frac{4 u}{r^{2}}=u \sum_{i} A_{i} \delta\left(r-r_{i}\right)
$$

In the intervals $r_{i}<r<r_{i+1}$, the right-hand side is zero and we can easily show that the appropriate vacuum solution for $u$ is $u=\tilde{a}_{i} r^{2}+\tilde{b}_{i} / r^{2}, \quad \tilde{a}_{i}$ and $\tilde{b}_{i}$ being functions of $\xi(i=1, \ldots, N-1)$. For $r<r_{1}$, the correct behavior is $u=\tilde{a}_{0} r^{2}+2 d / r^{2}$ (where $d=-q a$ is the negative dipole moment), while $u=\tilde{b}_{N} / r^{2}$ for $r>r_{N}$ $\left(\tilde{a}_{N}=0\right.$, so that $u \rightarrow 0$ when $\left.r \rightarrow \infty\right)$. The matching conditions for $u$ at $r=r_{i}$ are actually the same as those of (46), namely

$$
u\left(r_{i}^{+}, \xi\right)=u\left(r_{i}^{-}, \xi\right), \quad \partial_{r} u\left(r_{i}^{+}, \xi\right)-\partial_{r} u\left(r_{i}^{-}, \xi\right)=A_{i} u\left(r_{i}^{-}, \xi\right) .
$$

Given the analytical expression for $u$, these conditions yield

$$
\begin{gathered}
\tilde{a}_{i} r_{i}^{2}+\frac{\tilde{b}_{i}}{r_{i}^{2}}=\tilde{a}_{i-1} r_{i}^{2}+\frac{\tilde{b}_{i-1}}{r_{i}^{2}}, \\
2 \tilde{a}_{i} r_{i}-\frac{2 \tilde{b}_{i}}{r_{i}^{3}}=2 \tilde{a}_{i-1} r_{i}-\frac{2 \tilde{b}_{i-1}}{r_{i}^{3}}+A_{i}\left(\tilde{a}_{i-1} r_{i}^{2}+\frac{\tilde{b}_{i-1}}{r_{i}^{2}}\right), \\
\quad i=1, \ldots, N
\end{gathered}
$$

with $\tilde{b}_{0}=2 d$ and $\tilde{a}_{N}=0$. The above results can be cast into a recurrence relation:

$$
\left(\begin{array}{c}
\tilde{a}_{i} \\
\tilde{b}_{i}
\end{array}\right)=\tilde{M}_{i}\left(\begin{array}{c}
\tilde{a}_{i-1} \\
\tilde{b}_{i-1}
\end{array}\right)
$$

where the transfer matrix $\tilde{M}_{i}$ is 

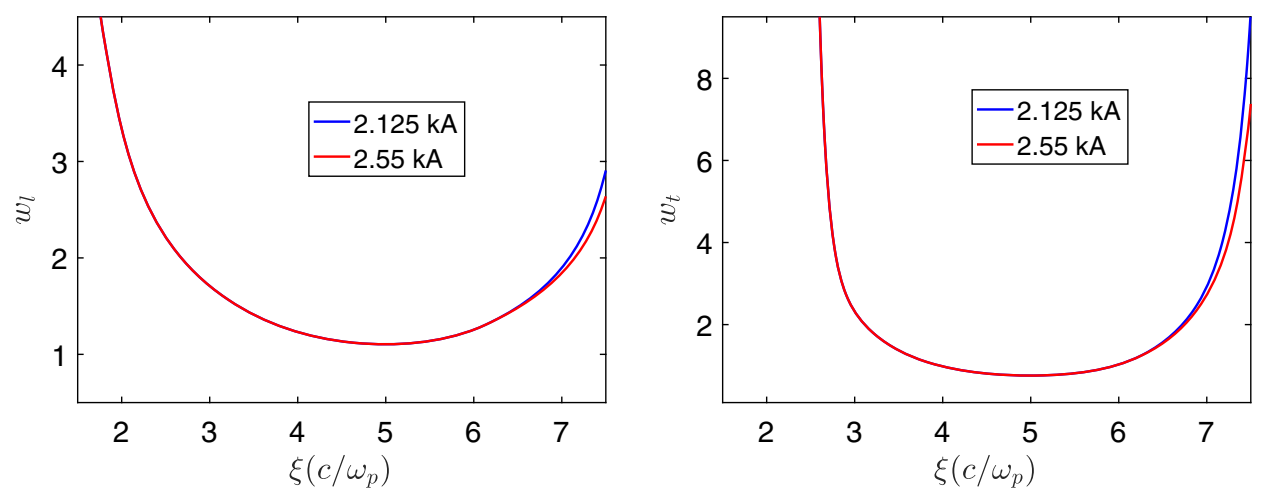

FIG. 4. Scaled longitudinal and transverse wakefields $w_{l}=4 \hat{M}_{12} / \hat{M}_{11}$ and $w_{t}=8 \tilde{M}_{12} / \tilde{M}_{11}$ as functions of $\xi$. The results shown here correspond to the parameter set used in the beam loading study of Sec. VI.

$$
\tilde{M}_{i}=\left(\begin{array}{cc}
1+\frac{1}{4} A_{i} r_{i} & \frac{1}{4 r_{i}^{3}} A_{i} \\
-\frac{1}{4} A_{i} r_{i}^{5} & 1-\frac{1}{4} A_{i} r_{i}
\end{array}\right)
$$

Combining these matrix manipulations, we obtain

$$
\left(\begin{array}{l}
\tilde{a}_{N} \\
\tilde{b}_{N}
\end{array}\right)=\tilde{M}\left(\begin{array}{l}
\tilde{a}_{0} \\
\tilde{b}_{0}
\end{array}\right)=\left(\begin{array}{ll}
\tilde{M}_{11} & \tilde{M}_{12} \\
\tilde{M}_{21} & \tilde{M}_{22}
\end{array}\right)\left(\begin{array}{c}
\tilde{a}_{0} \\
\tilde{b}_{0}
\end{array}\right)
$$

where $\tilde{M}=\tilde{M}_{N} \tilde{M}_{N-1} \cdots \tilde{M}_{1}$ is the new cumulative matrix. Recalling that $\tilde{b}_{0}=2 d$ and $\tilde{a}_{N}=0$, we obtain $\tilde{a}_{0}=-2 d \tilde{M}_{12} / \tilde{M}_{11}$. The coefficient $\tilde{a}_{0}$ has the following physical meaning: the transverse force per unit charge $F_{x}$ (scaled by $m c \omega_{p} / e$ ) acting on a trailing point charge at $\xi>\xi_{0}$ can be shown to be $F_{x}=-4 \tilde{a}_{0}\left(\xi-\xi_{0}\right)$. Thus, $\tilde{a}_{0}$ can be thought of as a measure of the transverse wake. A basic numerical illustration of the variation of the wakefields along the bubble is given in Fig. 4. Specifically, we plot the quantities $w_{l}=\Delta E_{z}(0, \xi) / \nu_{q}=4 \hat{M}_{12} / \hat{M}_{11}$ and $w_{t}=-4 \tilde{a}_{0} / d=8 \tilde{M}_{12} / \tilde{M}_{11}$ as functions of $\xi$ (we note that both $w_{l}$ and $w_{t}$ are independent of $q$ ). A more comprehensive study, including a comparison with some phenomenological formulas, can be found in [20].

In conclusion, we have shown that the short-range wakefields induced within the plasma bubble can be expressed in terms of matrix elements that can be calculated if the positions and momenta of the simulation macroparticles are known (recall that knowing the macroparticle coordinates is sufficient for determining the fields at a given $\xi)$. Thus, apart from obtaining the details of the plasma flow, running our algorithm leads directly to useful information about the transverse and longitudinal wakefields.

\section{CONCLUSIONS}

In this paper, we have developed a novel, steady-state simulation technique that can deal with an axisymmetric PWFA configuration in the blowout regime. In particular, we have studied the propagation of two ultrarelativistic, nonevolving and axially symmetric bunches of arbitrary density profile through a cold plasma of uniform density. After formulating and analyzing the single-particle equations of motion for the plasma electrons, we show how the nonlinear plasma flow of the PWFA can be modeled using simulation macroparticles. In order to obtain a self-consistent description of the interaction, we combine our analysis of the plasma dynamics with the equations that govern the formation of the electromagnetic fields. A crucial feature of our semianalytical treatment is that, given the macroparticle coordinates at a given longitudinal position within the plasma bubble, we can determine the full pattern of the fields at that particular location. This decoupling between the transverse and longitudinal directions is what makes our approach different from the existing numerical algorithms [13,17,19] and allows us to determine the structure of the bubble in a systematic and efficient way. Using our technique, we have studied the beam loading effect for a Gaussian driver/witness beam configuration. Our results regarding the dimensions of the plasma cavity and the electric field pattern that is established within it are in good agreement with those obtained from QuickPIC, a well-known particle-in-cell PWFA code.

Compared to such tools, our code differs in some key respects. To begin with, it does not utilize a grid (radial or Cartesian) in order to resolve the fields in the transverse plane. As a result, we need neither to deposit field and current values at the grid points nor to interpolate the fields at the macroparticle locations based on the former. We expect that this would make our algorithm free of many numerical issues that are inherent in a traditional PIC code. Moreover, in the current iteration of the code, the nonevolving external electron beam (driver and witness bunches) is modeled through an analytical technique and not by using macroparticles. This simplifying feature also implies that our approach can be extended to the case of a laser wakefield accelerator, at least for schemes with negligible laser beam depletion. Another possibility for the PWFA version of the code would be to add the neglected self-consistent evolution of the drive/witness beams as they propagate through the plasma. This 
modification would retain the quasistatic approximation but would require the introduction of drive macroparticles and time evolution, making the code considerably slower. Overall, we believe that our approach offers a simple and efficient way of studying plasma-based configurations with cylindrical symmetry that is an attractive alternative to existing codes, especially whenever versatility-and not computational sophistication-is the primary concern. The former feature (versatility) is particularly emphasized by the fact our algorithm can be used directly for the calculation of the short-range wakefields inside the plasma cavity, thus making it a very useful tool for stability-related studies.

\section{ACKNOWLEDGMENTS}

We would like to thank V. Khudik for his important contributions to this work. We are thankful to $\mathrm{X}$. Xu for providing the QUICKPIC data for the beam loading study and to W. An for the comparison of our algorithm with that of QuickPIC. This work was supported by the Department of Energy, Contract No. DE-AC03-76SF00515.

\section{APPENDIX: DEALING WITH DELTA FUNCTIONS AND THEIR DERIVATIVES IN THE EQUATION FOR $\bar{B}_{\theta}$}

In this Appendix, we study the mathematical properties of a driven equation similar to the one for $\bar{B}_{\theta}$ [see Eqs. (19), (22) and (23) in the main text]. To start with, let us consider the following model equation for the function $y(x)$ :

$$
\frac{d^{2} y(x)}{d x^{2}}=a y(x) \delta\left(x-x_{0}\right)+b \delta\left(x-x_{0}\right)+c_{0} \delta^{\prime}\left(x-x_{0}\right),
$$

where $x_{0}, a, b$ and $c_{0}$ are constants. Our goal is to obtain the matching conditions for $y$ and $y^{\prime}$ at $x=x_{0}$ [note that both variables are discontinuous at the position of the singularity, on account of the presence of the delta function derivative on the rhs of Eq. (A1)]. We first note that $\delta^{\prime}\left(x-x_{0}\right)=h^{\prime \prime}\left(x-x_{0}\right)$, where $h\left(x-x_{0}\right)$ is the unit step function ( 0 for $x<x_{0}$ and 1 for $x>x_{0}$ ) and rewrite the above equation as

$$
\frac{d^{2}\left[y(x)-c_{0} h\left(x-x_{0}\right)\right]}{d x^{2}}=[a y(x)+b] \delta\left(x-x_{0}\right) .
$$

Introducing $z(x)=y(x)-c_{0} h\left(x-x_{0}\right)$, we have

$$
\begin{aligned}
\frac{d^{2} z(x)}{d x^{2}} & =[a z(x)+b] \delta\left(x-x_{0}\right)+a c_{0} h\left(x-x_{0}\right) \delta\left(x-x_{0}\right) \\
& =[a z(x)+b] \delta\left(x-x_{0}\right)+\frac{1}{2} a c_{0} \frac{d}{d x} h^{2}\left(x-x_{0}\right) \\
& =\left[a z(x)+b+\frac{1}{2} a c_{0}\right] \delta\left(x-x_{0}\right),
\end{aligned}
$$

where we have used the relations $\delta\left(x-x_{0}\right)=h^{\prime}\left(x-x_{0}\right)$ and $h^{2}\left(x-x_{0}\right)=h\left(x-x_{0}\right)$. Integrating the above result from $x=x_{0}^{-}$to $x=x_{0}^{+}$, we obtain

$$
z^{\prime}\left(x_{0}^{+}\right)-z^{\prime}\left(x_{0}^{-}\right)=a z\left(x_{0}\right)+b+\frac{1}{2} a c_{0} .
$$

We note that $z(x)$ is continuous at $x=x_{0}$ [the rhs of Eq. (A3), as shown in the second line, does not contain a delta function derivative], so $z\left(x_{0}\right)=y\left(x_{0}^{-}\right)$is well defined. Collecting everything, we arrive at the matching conditions,

$$
\begin{aligned}
y\left(x_{0}^{+}\right) & =y\left(x_{0}^{-}\right)+c_{0}, \\
y^{\prime}\left(x_{0}^{+}\right)-y^{\prime}\left(x_{0}^{-}\right) & =a y\left(x_{0}^{-}\right)+b+\frac{1}{2} a c_{0} \\
& =\frac{1}{2} a\left[y\left(x_{0}^{+}\right)+y\left(x_{0}^{-}\right)\right]+b .
\end{aligned}
$$

When we have the operator $\partial_{x} x^{-1} \partial_{x}(x y)$ on the left-hand side of (A1), we instead need to consider the equation

$$
y^{\prime \prime}+\frac{1}{x} y^{\prime}-\frac{y}{x^{2}}=a y(x) \delta\left(x-x_{0}\right)+b \delta\left(x-x_{0}\right)+c_{0} \delta^{\prime}\left(x-x_{0}\right) .
$$

Equation (A3) is now replaced by

$$
\begin{aligned}
z^{\prime \prime} & +\frac{1}{x}\left[z^{\prime}+c_{0} \delta\left(x-x_{0}\right)\right]-\frac{z+c_{0} h\left(x-x_{0}\right)}{x^{2}} \\
& =[a z(x)+b] \delta\left(x-x_{0}\right)+\frac{1}{2} a c_{0} \frac{d}{d x} h^{2}\left(x-x_{0}\right) .
\end{aligned}
$$

Moving the term $c_{0} \delta\left(x-x_{0}\right) / x=c_{0} \delta\left(x-x_{0}\right) / x_{0}$ to the rhs, we have

$$
\begin{aligned}
z^{\prime \prime}+ & \frac{1}{x} z^{\prime}-\frac{z+c_{0} h\left(x-x_{0}\right)}{x^{2}} \\
= & {\left[a z(x)+b-c_{0} / x_{0}\right] \delta\left(x-x_{0}\right) } \\
& +\frac{1}{2} a c_{0} \frac{d}{d x} h^{2}\left(x-x_{0}\right) .
\end{aligned}
$$

Since $z$ is still continuous (no delta function derivatives among the driving terms), the added term proportional to $z^{\prime}$ does not affect the matching conditions at $x=x_{0}$. The same is true of the term proportional to the combination $z+c_{0} h\left(x-x_{0}\right)$, which has a finite discontinuity at $x=x_{0}$. Thus, the sole effect of the change in the form of the lhs of (A1) is the replacement of $b$ with $b-c_{0} / x_{0}$ in (A5).

By reviewing Eqs. (19), (22) and (23), we can easily verify that the equation for $\bar{B}_{\theta}$ is entirely analogous to the one studied above. In particular, the basic analogies are $x \rightarrow r, x_{0} \rightarrow r_{i}$ and $y \rightarrow \bar{B}_{\theta}$, so that we can use Eq. (A5) 
with

$$
\begin{aligned}
& y\left(x_{0}^{-}\right) \rightarrow a_{i-1} r_{i}+\frac{b_{i-1}}{r_{i}}, \quad y^{\prime}\left(x_{0}^{-}\right) \rightarrow a_{i-1}-\frac{b_{i-1}}{r_{i}^{2}}, \\
& y\left(x_{0}^{+}\right) \rightarrow a_{i} r_{i}+\frac{b_{i}}{r_{i}}, \quad y^{\prime}\left(x_{0}^{+}\right) \rightarrow a_{i}-\frac{b_{i}}{r_{i}^{2}}
\end{aligned}
$$

and $a \rightarrow A_{i}, b \rightarrow B_{i}$ and $c_{0} \rightarrow C_{i}$. This leads directly to Eq. (25) in the main text.

[1] I. Blumenfeld, C. E. Clayton, F.-J. Decker, M. J. Hogan, C. Huang, R. Ischebeck, R. Iverson, C. Joshi, T. Katsouleas, N. Kirby, W. Lu, K. A. Marsh, W. B. Mori, P. Muggli, E. Oz, R. H. Siemann, D. Walz, and M. Zhou, Energy doubling of $42 \mathrm{GeV}$ electrons in a metre-scale plasma wakefield accelerator, Nature (London) 445, 741 (2007).

[2] M. Litos et al., High-efficiency acceleration of an electron beam in a plasma wakefield accelerator, Nature (London) 515, 92 (2014).

[3] R. D. Ruth, A. W. Chao, P. L. Morton, and P. B. Wilson, A plasma wake field accelerator, Part. Accel. 17, 171 (1985).

[4] P. Chen, J. M. Dawson, R. W. Huff, and T. Katsouleas, Acceleration of Electrons by the Interaction of a Bunched Electron Beam with a Plasma, Phys. Rev. Lett. 54, 693 (1985).

[5] K. L. Bane, P. Chen, and P. B. Wilson, in Proceedings of the 1985 Particle Accelerator Conference (PAC1985): Accelerator Engineering and Technology Vancouver, BC, 1985 [IEEE Trans. Nucl. Sci. 32, 3524 (1985)].

[6] J. B. Rosenzweig, B. Breizman, T. Katsouleas, and J. J. Su, Acceleration and focusing of electrons in two-dimensional nonlinear plasma wake fields, Phys. Rev. A 44, R6189 (1991).

[7] A. Pukhov and J. Meyer-ter Vehn, Laser wake field acceleration: the highly non-linear broken-wave regime, Appl. Phys. B 74, 355 (2002).

[8] I. Kostyukov, A. Pukhov, and S. Kiselev, Phenomenological theory of laser-plasma interaction in "bubble" regime, Phys. Plasmas 11, 5256 (2004).
[9] W. Lu, C. Huang, M. Zhou, M. Tzoufras, F. S. Tsung, W. B. Mori, and T. Katsouleas, A nonlinear theory for multidimensional relativistic plasma wave wakefields, Phys. Plasmas 13, 056709 (2006).

[10] S. A. Yi, V. Khudik, C. Siemon, and G. Shvets, Analytic model of electromagnetic fields around a plasma bubble in the blow-out regime, Phys. Plasmas 20, 013108 (2013).

[11] G. Stupakov, B. Breizman, V. Khudik, and G. Shvets, Wake excited in plasma by an ultrarelativistic pointlike bunch, Phys. Rev. Accel. Beams 19, 101302 (2016).

[12] C. Nieter and J. R. Cary, VORPAL: a versatile plasma simulation code, J. Comput. Phys. 196, 448 (2004).

[13] K. V. Lotov, Fine wakefield structure in the blowout regime of plasma wakefield accelerators, Phys. Rev. ST Accel. Beams 6, 061301 (2003).

[14] W. B. Mori, Recent advances and some results in plasmabased accelerator modeling, AIP Conf. Proc. 647, 11 (2002).

[15] T. Mehrling, C. Benedetti, C. B. Schroeder, and J. Osterhoff, HiPACE: a quasi-static particle-in-cell code, Plasma Phys. Controlled Fusion 56, 084012 (2014).

[16] A. Burov, V. Lebedev, and S. Nagaitsev, Instability of a witness bunch in a plasma bubble, arXiv:1602.05260.

[17] P. Mora and T. M. Antonsen, Jr., Kinetic modeling of intense, short laser pulses propagating in tenuous plasmas, Phys. Plasmas 4, 217 (1997).

[18] W. An, V. K. Decyk, W. B. Mori, and T. M. Antonsen, An improved iteration loop for the three dimensional quasistatic particle-in-cell algorithm: QuickPIC, J. Comput. Phys. 250, 165 (2013).

[19] C. Huang, V. Decyk, C. Ren, M. Zhou, W. Lu, W. Mori, J. Cooley, T. Antonsen, and T. Katsouleas, QUICKPIC: A highly efficient particle-in-cell code for modeling wakefield acceleration in plasmas, J. Comput. Phys. 217, 658 (2006).

[20] G. Stupakov, Short-range wakefields generated in the blowout regime of plasma-wakefield acceleration, arXiv: 1710.07371.

[21] N. Barov, J. B. Rosenzweig, M. C. Thompson, and R. B. Yoder, Energy loss of a high-charge bunched electron beam in plasma: Analysis, Phys. Rev. ST Accel. Beams 7, 061301 (2004). 